University of Wollongong

Research Online

Faculty of Engineering - Papers (Archive)

Faculty of Engineering and Information

Sciences

March 1991

\title{
Fermi-energy-edge singularity in quantum wells containing more than one occupied subband
}

\author{
M. S. Skolnick \\ Royal Signals and Radar Establishment, UK \\ D. M. Whittaker \\ Royal Signals and Radar Establishment, UK \\ Philip E. Simmonds \\ University of Wollongong, simmonds@uow.edu.au \\ T. A. Fisher \\ University of Wollongong \\ M. K. Saker \\ Royal Signals and Radar Establishment, UK
}

See next page for additional authors

Follow this and additional works at: https://ro.uow.edu.au/engpapers

Part of the Engineering Commons

https://ro.uow.edu.au/engpapers/273

\section{Recommended Citation}

Skolnick, M. S.; Whittaker, D. M.; Simmonds, Philip E.; Fisher, T. A.; Saker, M. K.; Rorison, J. M.; Smith, R. S.; Kirby, P. B.; and White, C. R.: Fermi-energy-edge singularity in quantum wells containing more than one occupied subband 1991.

https://ro.uow.edu.au/engpapers/273

Research Online is the open access institutional repository for the University of Wollongong. For further information contact the UOW Library: research-pubs@uow.edu.au 


\section{Authors}

M. S. Skolnick, D. M. Whittaker, Philip E. Simmonds, T. A. Fisher, M. K. Saker, J. M. Rorison, R. S. Smith, P. B. Kirby, and C. R. White 


\title{
Fermi-energy-edge singularity in quantum wells containing more than one occupied subband
}

\author{
M. S. Skolnick and D. M. Whittaker \\ Royal Signals and Radar Establishment, St. Andrews Road, Malvern, Worcs WR14 3PS, United Kingdom \\ P. E. Simmonds \\ Royal Signals and Radar Establishment, St. Andrews Road, Malvern, Worcs WRI4 3PS, United Kingdom \\ and Department of Physics, University of Wollongong, Wollongong, New South Wales, Australia 2500
}

\section{T. A. Fisher}

Department of Physics, University of Wollongong, Wollongong, New South Wales, Australia 2500

\author{
M. K. Saker and J. M. Rorison \\ Royal Signals and Radar Establishment, St. Andrews Road, Malvern, Worcs WR14 3PS, United Kingdom \\ R. S. Smith and P. B. Kirby \\ GEC Hirst Research Centre, Wembley, Middlesex HA9 7PP, United Kingdom \\ C. R. H. White \\ Department of Physics, University of Nottingham, Nottingham, United Kingdom
}

(Received 10 December 1990)

\begin{abstract}
A study of the Fermi-energy-edge singularity in asymmetric modulation-doped quantum wells is reported. It is shown that high densities of free carriers in filled $n=1$ electron subbands have no significant influence on the edge singularity in partly occupied $n=2$ subbands, demonstrating the weakness of intersubband Coulomb-scattering processes. The enhancement of oscillator strength at the Fermi energy is of very similar magnitude to that for excitonic transitions in undoped quantum wells.
\end{abstract}

Modulation-doped quantum wells (QW's) provide nearly ideal systems for the study of many-electron effects such as band-gap renormalization, ${ }^{1,2}$ the Fermi-energyedge singularity (FEES), ${ }^{3-8}$ and shake-up processes. ${ }^{9}$ The FEES is manifested at high electron density $\left(n_{s}\right)$ as an enhancement of oscillator strength (the Mahan exciton $^{10}$ ) for optical absorption [photoluminescence (PL)] transitions occurring just above (below) the electron Fermi level $\left(E_{F}\right)$. In optical absorption, the FEES arises from the collective Coulomb interaction between the photocreated electron-hole pair and the Fermi sea of electrons. ${ }^{10-14}$ One of the characteristic features of this many-body interaction is its very strong temperature dependence; at temperatures where the broadening $(\sim k T)$ of the Fermi surface is of the order of the exciton binding energy, the spectral region of enhanced oscillator strength is strongly broadened and weakened. ${ }^{11,13,14}$

In the present paper, a study of the temperature dependence of the "excitonic" features occurring at $E_{F}$ in both PL and PL-excitation (PLE) spectroscopy is reported in a series of asymmetric, modulation-doped $\mathrm{Al}_{y}, \mathrm{Ga}_{1-y}$ As
$(y=0.23)-\operatorname{In}_{x} \mathrm{Ga}_{1-x} \mathrm{As}(x=0.07-0.11)$-GaAs strainedlayer QW's. ${ }^{15-17}$ The relatively wide QW's (150-250- $\AA$ width) cover the range from electron Fermi energy $\varepsilon_{F n}$ $=E_{F}-E_{n}$ greater than $E_{2}-E_{1}$, the spacing between the first two $(n=1,2)$ electron subbands, close to, but just less than $E_{2}-E_{1}$, and much less than $E_{2}-E_{1}$. This enables the effects on the oscillator strength at $E_{F}$, of high densities of carriers $\left(n_{s} \sim 10^{12} \mathrm{~cm}^{-2}\right)$ distributed between two subbands, to be studied.

The samples were grown ${ }^{16}$ by molecular-beam epitaxy and consisted of the following layers grown on a semiinsulating GaAs substrate: $1-\mu \mathrm{m}$ undoped GaAs, $\mathrm{In}_{x} \mathrm{Ga}_{1-x}$ As quantum well (thickness $d$ ), undoped $\mathrm{Al}_{y} \mathrm{Ga}_{1-y}$ As $(y=0.23) \quad$ spacer (thickness $\left.w_{1}\right)$, $\mathrm{Al}_{1}, \mathrm{Ga}_{1-1}$ As doped region (thickness $w_{2}$, doping level $n_{2}$ ), and GaAs capping layer $\left(w_{3}, n_{3}\right)$. Details are given in Table I, together with $\left(E_{2}-E_{1}\right)$ and $E_{F}$ values determined from magneto-PL and Shubnikov-de Haas ( $\mathrm{SdH})$ studies. ${ }^{18} \mathrm{PL}$ and PLE were carried out from 2 to $50 \mathrm{~K}$, in the low-intensity limit using $\sim 10 \mathrm{~mW} / \mathrm{cm}^{2}$ of radiation from a Styryl 9 dye laser, and detected using a Ge photo-

TABLE I. Sample details.

\begin{tabular}{|c|c|c|c|c|c|c|c|c|c|}
\hline Sample & $\begin{array}{c}d \\
(\AA)\end{array}$ & $\begin{array}{l}w_{1} \\
(\AA)\end{array}$ & $\begin{array}{l}w_{2} \\
(\AA)\end{array}$ & $\begin{array}{c}n_{2} \\
\left(\mathrm{~cm}^{-3}\right)\end{array}$ & $\begin{array}{l}w_{3} \\
(\AA)\end{array}$ & $\begin{array}{c}n_{3} \\
\left(\mathrm{~cm}^{-3}\right)\end{array}$ & $\begin{array}{c}E_{F}-E_{1} \\
(\mathrm{meV})\end{array}$ & $\begin{array}{c}E_{2}-E_{1} \\
(\mathrm{meV})\end{array}$ & $x$ \\
\hline$A$ & 250 & 100 & 250 & $1.5 \times 10^{18}$ & 200 & $1 \times 10^{14}$ & 30.5 & 27.0 & $0.10(5)$ \\
\hline$B$ & 200 & 40 & 400 & $1 \times 10^{18}$ & 100 & $2 \times 10^{18}$ & 34.0 & 34.5 & $0.07(5)$ \\
\hline$C$ & 150 & 100 & 250 & $1.5 \times 10^{18}$ & 200 & $1 \times 10^{14}$ & 27.5 & 43.0 & $0.09(0)$ \\
\hline
\end{tabular}


diode or GaAs photomultiplier.

The PL spectrum $(2 \mathrm{~K})$ for sample $A$ is shown in Fig. 1 (dotted curve). It consists of two bands, labeled $E_{21}$ and $E_{11}$, arising from recombination of $n=2$ and 1 electrons, respectively, with holes in the $n=1$ valence subband. This sample with $d=250 \AA$ has $E_{2}-E_{1}=27.0 \mathrm{meV}$, and $\varepsilon_{F 1}=30.5 \mathrm{meV}$, so that $E_{F}$ resides $3.5 \mathrm{meV}(n=2$ density $n_{s}^{2}$ of $1 \times 10^{11} \mathrm{~cm}^{-2}$ ) above the bottom of the $n=2$ subband. $E_{21}$ shows Landau-level (LL) splitting in magnetic field, characteristic of an $\varepsilon_{F 2}$ of $3.5 \mathrm{meV} .^{18}$

The peak height for $E_{21}$ transitions is 22 times greater than that for $E_{11}$. The principal reason is the much greater overlap for electrons in the $n=2$ subband with $n=1$ valence-band holes, than for those in $n=1$. The inset to Fig. 1 shows the band-edge profiles, and $n=2$ and 1 wave functions $\left(\psi_{2}^{e}, \psi_{1}^{e}\right.$, and $\left.\psi_{1}^{h}\right)$, obtained from a selfconsistent (Hartree) solution of Poisson's and Schrödinger's equation. The band-bending potential in these wide QW's causes a strong modification of the wave functions compared to $n_{s}=0$, and leads to very marked changes in the oscillator strengths for interband transitions. ${ }^{2,15,17,19} \psi_{1}^{e}$ is well-confined towards the $\mathrm{Al}_{y^{\prime}}$ $\mathrm{Ga}_{1-y} \cdot \mathrm{As}-\mathrm{In}_{x} \mathrm{Ga}_{1-x} \mathrm{As}$ interface whereas $\psi_{2}^{e}$ is much more extended, but with larger amplitude towards the $\mathrm{In}_{x} \mathrm{Ga}_{1-x} \mathrm{As}-\mathrm{GaAs}$ interface. $\psi_{1}^{h}$ is strongly confined towards the $\mathrm{In}_{x} \mathrm{Ga}_{1-x}$ As-GaAs interface. Using the wave functions shown in the inset to Fig. 1, a ratio $\left(r_{c}\right)$ of 11 is calculated for the electron-hole overlap integrals squared for the $E_{21}$ to $E_{11}$ transitions, as compared to the experimental ratio $\left(r_{e}\right)$ of intensities of $22 .{ }^{20}$

Such a calculation allows an estimate to be made for the magnitude of any excitonic enhancement of the $E_{21}$ oscillator strength. Since $E_{21}$ transitions occur close to the Fermi energy $(<3 \mathrm{meV})$, a marked enhancement of oscillator strength is expected. For $E_{11}$ transitions excitonic interactions are expected to be very weak. The $E_{11}$ recombination peaks at the bottom of the band, since the

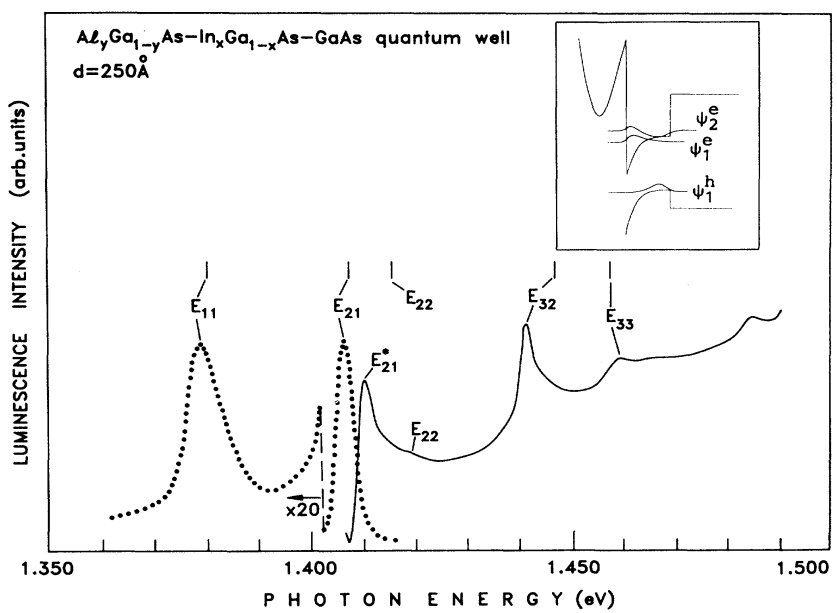

FIG. 1. PL (dotted) and PLE (solid line) spectra for sample $A(d=250 \AA)$ at $2 \mathrm{~K}$. The PL spectrum is composed of $E_{11}$ and $E_{21}$ recombination bands. In PLE, $E_{21}^{*}, E_{22}, E_{32}$, and $E_{33}$ absorption features are observed. The vertical lines above the spectrum are theoretical energies. Inset: self-consistent quantum well potential and wave functions. only transitions allowed by wave-vector conservation arise for electrons at $E_{1} \approx 0(k=0)$, as expected for a $\mathrm{QW}$ with weak disorder. ${ }^{3,14}$ For the excitonic interaction to take place electron-hole scattering processes must occur into empty electron states. The only unoccupied electron states are above $E_{F}$, thus leading to very weak excitonic effects for the $k \approx 0 E_{11}$ transitions, which are also weak due to the small $\psi_{1}^{e}, \psi_{1}^{h}$ overlap.

Comparison of the experimental $\left(r_{e}=22\right)$ and theoretical $\left(r_{c}=11\right)$ ratios leads to a value for the excitonic enhancement of $E_{21}$ transitions of $\sim 2( \pm 0.6) .{ }^{20}$ This is a very reasonable value for the magnitude of excitonic effects, and is close to that found in absorption experiments on $250-\AA \AA$ undoped QW's having similar exciton linewidths $(3.5 \mathrm{meV}) .^{21}$ This conclusion is supported by the PLE, where possible complications due to population effects are eliminated. We have obtained similar values for the excitonic enhancement for $E_{21}$ transitions in a variety of asymmetric QW's. We find no evidence for anomalous enhancements of oscillator strengths at $E_{F}$, as reported in finite magnetic fields, ${ }^{17}$ so long as the dominant effects of the band-bending potential on the wave functions are taken into account.

The PLE spectrum for sample $A$ is shown by the solid curve in Fig. 1. It consists of a sharp "excitonic" peak at $E_{F}$ (labeled $E_{21}^{*}$ ) corresponding to transitions into empty states in the $n=2$ subband. Transitions between higher subbands are also observed. The vertical lines (marked $E_{11}, E_{21}$, etc.) are calculated transition energies (as in Figs. 3 and 4). They are in good agreement with experiment, further supporting the calculations of the oscillator strengths. The $x$ values of the $\operatorname{In}_{x} \mathrm{Ga}_{1-x}$ As are fitting parameters (see Table I) in the transition-energy calculations, after allowance of $15 \mathrm{meV}$ for the band-gap renormalization at $n_{s}=9 \times 10^{11} \mathrm{~cm}^{-2} .^{2}$ The excitonic peak at $E_{21}^{*}$ is enhanced by a factor of $\sim 2$ relative to its continuum, in agreement with the deduction of $\sim 2$ enhancement from the comparison of the $E_{21}, E_{11}$ PL intensities.

The temperature dependence of the PL ( $E_{21}$ only) and PLE for sample $A$ at 2,21 , and $38 \mathrm{~K}$ is shown in Figs. 2(a)-2(c). At $2 \mathrm{~K}$ [Fig. 2(a)] the PL shows the asymmetric line shape characteristic of an $\varepsilon_{F}=3.5 \mathrm{meV}$. With increasing temperature [Figs. 2(b) and 2(c)] this broadens to higher energy showing the Boltzmann tail expected at 21 and $38 \mathrm{~K}$, respectively. The PLE spectra for $E_{21}^{*}$ show a very strong temperature dependence. The sharp $E_{21}^{*}$ feature at $2 \mathrm{~K}$ (full width at half maximum 3.3 $\mathrm{meV}$ ) is strongly broadened at $21 \mathrm{~K}$ (width $11 \mathrm{meV}$ ) and is visible only weakly at $38 \mathrm{~K}$.

This behavior is characteristic of excitonic features at $E_{F}$ (FEES) and arises when the thermal excitations $(\sim k T)$ are of the same order as the Coulomb interaction energy between the photoexcited electron-hole pair and the Fermi sea of electrons. ${ }^{11,13,14}$ The Coulomb interaction energy is expected to be of the order of the exciton binding energy, $\sim 5 \mathrm{meV}$ for the present wide $\mathrm{In}_{0.1}$ $\mathrm{Ga}_{0.9}$ As QW. ${ }^{22}$ By contrast the exciton feature $E_{32},-30$ meV above $E_{F}$ broadened only by $\mathrm{LO}$ phonon ionization processes, shows very little temperature dependence, in agreement with previous studies. ${ }^{4-6}$ The present results show that a very strong temperature dependence of the ex- 


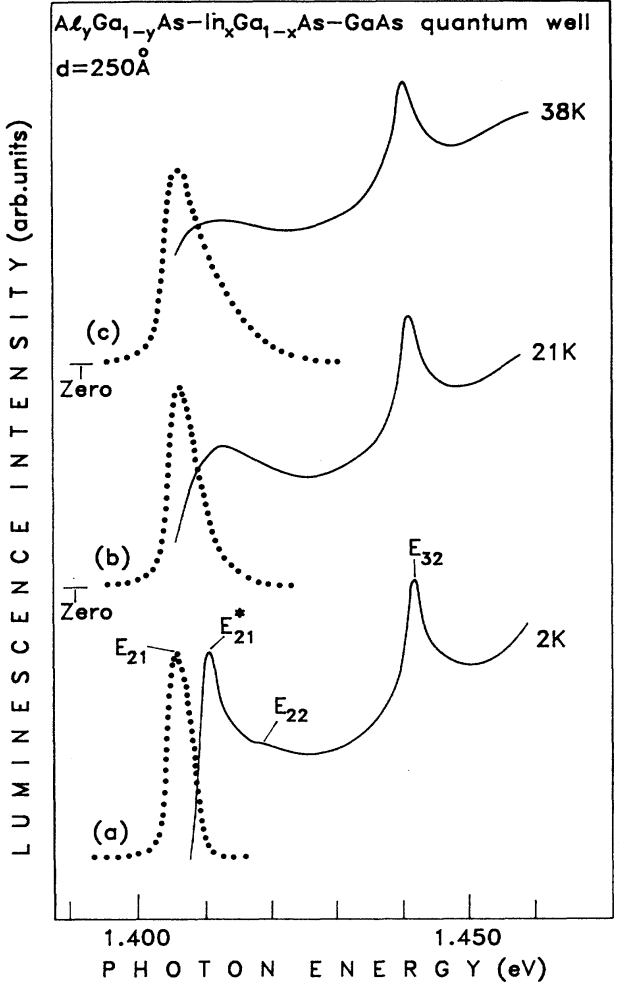

FIG. 2. Temperature dependence of PL (dotted) and PLE (solid line) spectra for sample $A$ at 2,21 , and $38 \mathrm{~K}$ in (a), (b), and (c). The presence of $1 \times 10^{11} \mathrm{~cm}^{-2}$ free carriers in the $n=2$ electron subband gives rise to the very strong temperature dependence of the $E_{21}^{*}$ feature at $E_{F}$.

citonic feature at $E_{F}$ is obtained even for a Fermi energy in the $n=2$ subband of only $3.5 \mathrm{meV}$, consistent with the results of Ref. 6 for a populated $n=1$ subband.

Figure 3(a) shows the 2-K PL and PLE for the 200- $\AA$ wide sample $B$, where $E_{F}$ resides close to the bottom of $E_{2}$ (within $\pm 1 \mathrm{meV}$, Table I). Both $E_{21}$ and $E_{11}$ PL is observed, although in this case no LL splitting, or narrowing, of $E_{21}$ is observed in magnetic field, allowing an upper limit of $\sim 1.0 \mathrm{meV}$ to be placed on $\varepsilon_{F 2}\left(n_{s}^{2} \leq 3 \times 10^{10}\right.$ $\mathrm{cm}^{-2}$ ), in $n=2$. Even though the population of $E_{2}$ is small, $E_{21}$ is observed with its full oscillator strength relative to $E_{11}$; increasing the laser power by an order of magnitude causes no detectable change in the value of $r_{e}$ of 13 . This compares with a calculated ratio of $r_{c}=5.8$, and leads to a deduced value of 2.2 for the excitonic enhancement of the $E_{21}$ peak, close to that found for sample $A$. The analysis is strongly supported by the fact that the decreasing trend in $r_{e}$ with decreasing well width is well accounted for by the $r_{c}$ calculations (i.e., $r_{e}=22,13$ and $r_{c}=11,5.8$ for the 250- and 200- $\AA$ QW's, respectively).

The PLE spectrum [Fig. 3(b)] at $40 \mathrm{~K}$ shows little change relative to its form at $2 \mathrm{~K}$. A small decrease of the enhancement at $E_{21}$ of $\sim 0.7$ is observed from 2 to $40 \mathrm{~K}$ in very marked contrast to sample $A\left(n_{s}^{1}=7.8 \times 10^{11} \mathrm{~cm}^{-2}\right.$, $n_{s}^{2}=1 \times 10^{11} \mathrm{~cm}^{-2}$; sample $B, n_{s}^{1}=9.8 \times 10^{11} \mathrm{~cm}^{-2}$, $n_{s}^{2} \leq 3 \times 10^{10} \mathrm{~cm}^{-2}$ ) where excitonic effects at $E_{21}$ are barely visible at $40 \mathrm{~K} .^{23}$ This demonstrates clearly that

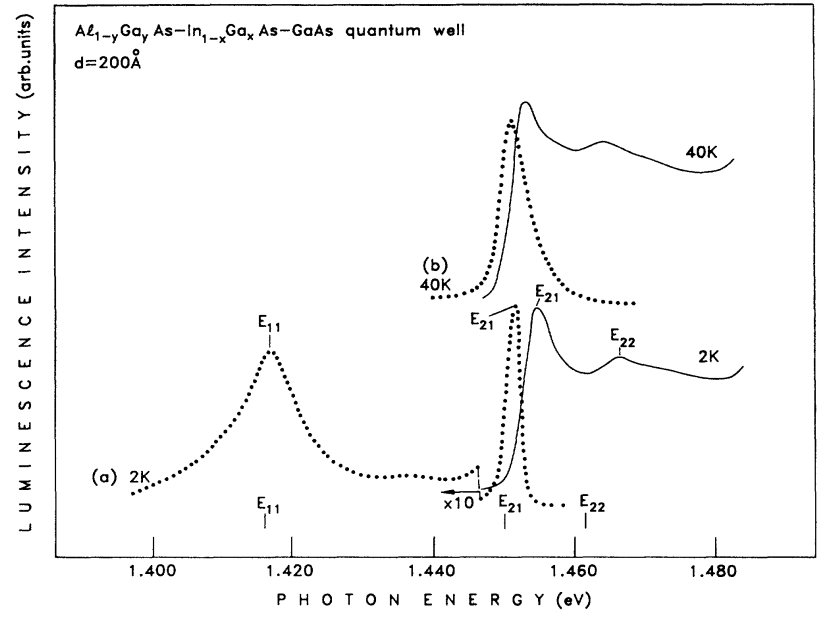

FIG. 3. PL (dotted) and PLE (solid line) spectra for sample

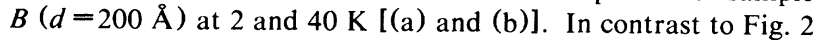
the excitonic feature close to $E_{F}\left(E_{21}\right)$ shows very little temperature dependence, due to the very small carrier density $\left(<3 \times 10^{10} \mathrm{~cm}^{-2}\right)$ in the $n=2$ subband.

only carriers in the subband where the excitonic peak occurs contribute to the quenching of the enhancement at elevated temperature. The presence of carriers at nearly the same energy in the $n=1$ subband has little or no effect on the temperature dependence of the excitonic peak observed for transitions into the $n=2$ subband. This arises since the carriers at $E_{F}$ in $n=1$ have a large wave vector

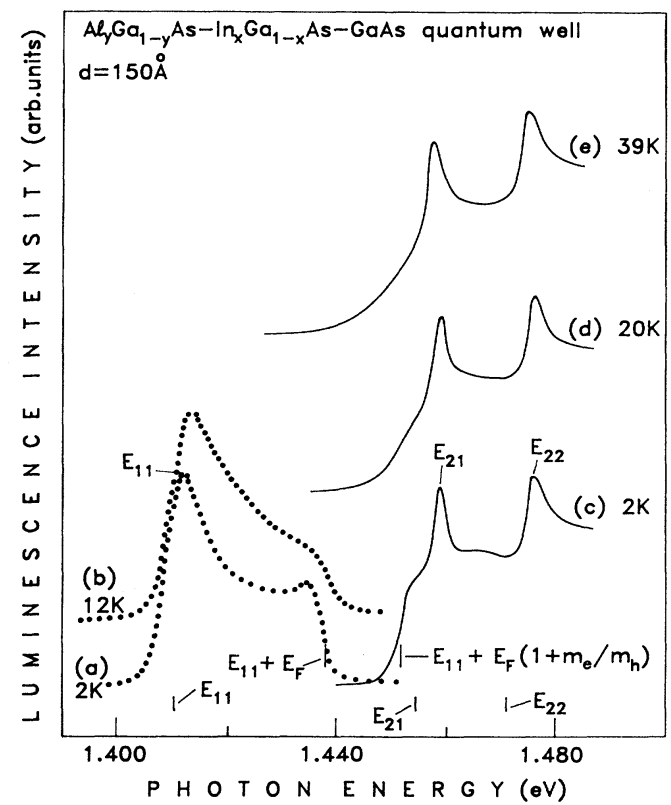

FIG. 4. PL (dotted) and PLE (solid line) spectra for sample $C(d=150 \AA)$. PL from the $n=1$ electron subband only is observed $\left(E_{F}=27.5 \mathrm{meV}, E_{2}-E_{1}=43 \mathrm{meV}\right)$. The very weak enhancement at $E_{11}+E_{F}\left(1+m_{e} / m_{h}\right)$ observed in (a) $(2 \mathrm{~K})$ has been quenched by (b) $12 \mathrm{~K}$. 
$\left(k_{F} \sim 2.5 \times 10^{6} \mathrm{~cm}^{-1}\right)$ and so will be coupled only weakly by the Coulomb interaction to the $n=2$ states at $k \approx 0$ which contribute to the observed transition. This contrasts with the $n=2$ electrons which can couple to the photo-excited electron-hole pair by a scattering process across $E_{F}$ with almost no change in $k$. The intersubband coupling will also be reduced by the orthogonality of the $n=2,1$ wave functions. ${ }^{24}$

In Fig. 4(a) the 2-K PL spectrum for sample $C$ $\left(d=150 \AA\right.$ ) is shown. $E_{F}$ is $15.5 \mathrm{meV}$ below $E_{2}$ (Table I), and only $E_{11} \mathrm{PL}$ is observed. This spectrum is taken with photomultiplier tube (PMT) detection which enhances features at $E_{F}(1.437 \mathrm{eV})$ by a factor of $10 \mathrm{com}$ pared to PL at the bottom of the band, due to the decreasing PMT response to lower energy. The PL spectra in Figs. 1, 3, and 4 are taken with the Ge detector whose response is flat over the region of interest. The PL spectrum in Fig. $4(\mathrm{a})$ peaks at $E_{11}$ and decreases towards $E_{F}$. A small enhancement is observed at $E_{F}$ before decreasing abruptly to higher energy. This weak enhancement is ascribed to a FEES (a similar, very weak effect was seen in Ref. 15). The degree of disorder (or hole localization) is just sufficiently great to permit observation of an enhancement at $E_{F}$. $^{3.14}$ In accordance with the very weak enhancement, and hence very weak "binding energy," it is extremely temperature sensitive. Raising the temperature to only $12 \mathrm{~K}$ [Fig. 4(b)] removes the enhancement, consistent with a binding energy of only $\sim 1.5 \mathrm{meV}$. PLE spectra at 2, 20, and $39 \mathrm{~K}$ are shown in Figs. 4(c)-4(e), respectively. The onset of PLE is upshifted by $\Delta E=12 \pm 0.5 \mathrm{meV}$ above $E_{F}$ in PL, due to the requirement for $k$ conservation in absorption. ${ }^{2,6,7} \Delta E=\left(m_{e}\right)$ $\left.m_{h}\right) E_{F}=12.5 \mathrm{meV}$ for $m_{e}=0.068 m_{o}$ and $m_{h}=0.15 m_{o},{ }^{25}$ in good agreement with experiment. An excitonic peak is not observed in PLE at $E_{11}+E_{F}\left(1+m_{e} / m_{h}\right)$, due to broadening by indirect transitions at large $k_{F}^{e} \cdot{ }^{6,12,14}$ With increasing temperature the $E_{11} \mathrm{PL}$ transitions show the expected broadening of the Fermi level, having a width of $\sim 2 \mathrm{kT}(8 \mathrm{meV})$ at $39 \mathrm{~K}$ [Fig. 4(e)].

In conclusion, the temperature dependence of the Fermi-energy-edge singularities in QW's containing more than one populated subband has been shown to be controlled by the carrier density in the subband in which the excitonic enhancement occurs. This is consistent with the relatively weak Coulomb coupling of states of widely differing wave vector. The strength of the excitonic enhancement at $E_{F}$ in structures with low electron density in the $n=2$ subband is of similar magnitude to that found for excitonic transitions in undoped QW's.

We would like to thank K. J. Nash for very helpful discussions.
${ }^{1}$ A. Pinczuk, J. Shah, R. C. Miller, A. C. Gossard, and W. Wiegmann, Solid State Commun. 50, 735 (1984).

${ }^{2}$ C. Delalande, G. Bastard, J. Orgonasi, J. A. Brum, H. W. Liu, M. Voos, G. Weimann, and W. Schlapp, Phys. Rev. Lett. 59, 2690 (1987).

${ }^{3}$ M. S. Skolnick, J. M. Rorison, K. J. Nash, D. J. Mowbray, P. R. Tapster, S. J. Bass, and A. D. Pitt, Phys. Rev. Lett. 58, 2130 (1987).

${ }^{4}$ J. S. Lee, Y. Iwasa, and N. Miura, Semicond. Sci. Technol. 2, 675 (1987).

${ }^{5}$ G. Livescu, D. A. B. Miller, D. S. Chemla, M. Ramaswamy, T. Y. Chang, N. Sauer, A. C. Gossard, and J. H. English, IEEE J. Quantum Electron. QE-24, 1677 (1988).

${ }^{6}$ M. K. Saker, M. S. Skolnick, P. A. Claxton, J. S. Roberts, and M. J. Kane, Semicond. Sci. Technol. 3, 691 (1988).

${ }^{7}$ H. Yoshimura, G. E. W. Bauer, and H. Sakaki, Phys. Rev. B 38, 10791 (1988).

${ }^{8}$ H. Kalt, K. Leo, R. Cingolani, and K. Ploog, Phys. Rev. B 40, 12017 (1989).

${ }^{9}$ R. Sooryakumar, D. S. Chemla, A. Pinczuk, A. C. Gossard, W. Wiegmann, and L. J. Sham, Solid State Commun. 54, 859 (1985).

${ }^{10}$ G. D. Mahan, Phys. Rev. 153, 882 (1967).

"S. Schmitt-Rink, C. Ell, and H. Haug, Phys. Rev. B 33, 1183 (1986).

${ }^{12}$ A. E. Ruckenstein and S. Schmitt-Rink, Phys. Rev. B 35, 7551 (1987).

${ }^{13}$ K. Ohtaka and Y. Tanabe, Phys. Rev. B 39, 3054 (1989).

${ }^{14}$ T. Uenoyama and L. J. Sham, Phys. Rev. Lett. 65, 1048 (1990).

${ }^{15}$ C. Colvard, N. Nouri, H. Lee, and D. Ackley, Phys. Rev. B 39, 8033 (1989).
${ }^{16}$ P. B. Kirby, J. A. Constable, and R. S. Smith, Phys. Rev. B 40, 3013 (1989).

${ }^{17}$ W. Chen, M. Fritze, A. V. Nurmikko, D. Ackley, C. Colvard, and H. Lee, Phys. Rev. Lett. 64, 2434 (1990).

${ }^{18}$ P. E. Simmonds, M. S. Skolnick, T. A. Fisher, C. R. H. White, R. S. Smith, and P. B. Kirby (unpublished).

${ }^{19}$ M. H. Meynadier, J. Orgonasi, C. Delalande, J. A. Brum, G. Bastard, M. Voos, G. Weimann, and W. Schlapp, Phys. Rev. B 34, 2482 (1986)

${ }^{20} r_{c}$ is a very sensitive function of well width and carrier density. For example, for $d=275 \AA, E_{F}=27.8 \mathrm{meV}, r_{c}$ is increased to $13.4\left(r_{c}=11.3\right.$ at $\left.d=250 \AA, E_{F}=31.2 \mathrm{meV}\right)$, whereas for $E_{F}=27.8 \mathrm{meV}$ and $d=250 \AA, r_{c}=8$. Thus, the $r_{c}$ values should be considered to have an uncertainty of $\approx \pm 30 \%$.

${ }^{21}$ Y. Masumoto, M. Matsuura, S. Tarucha, and H. Okamoto, Phys. Rev. B 32, 4275 (1985)

${ }^{22}$ Karen J. Moore, Geoffrey Duggan, Karl Woodbridge, and Christine Roberts, Phys. Rev. B 41, 1090 (1990).

${ }^{23}$ For $E_{F} 1 \mathrm{meV}$ below the $n=2$ subband, even at $40 \mathrm{~K}$ the $n=2$ population will be only $\sim 3 \times 10^{10} \mathrm{~cm}^{-2}$ over an energy range of $\mathrm{kT}$.

${ }^{24}$ J. F. Müller, A. E. Ruckenstein, and S. Schmitt-Rink (unpublished). In an analysis of the results of Ref. 17, these authors predict a strong enhancement of oscillator strength for transitions at $E_{F}$ in the $E_{1}$ subband, due to $E_{1}, E_{2}$ intersubband Coulomb scattering processes. However, the absence of a strong temperature dependence for transitions at $E_{F}$, unless $n_{s}^{2} \gtrsim 1 \times 10^{11} \mathrm{~cm}^{-2}$, in the present work shows that such intersubband coupling processes are relatively weak.

${ }^{25}$ E. D. Jones, S. K. Lyo, I. J. Fritz, J. F. Klem, J. E. Schirber, C. P. Tigges, and T. J. Drummond, Appl. Phys. Lett. 54, 2227 (1989). 